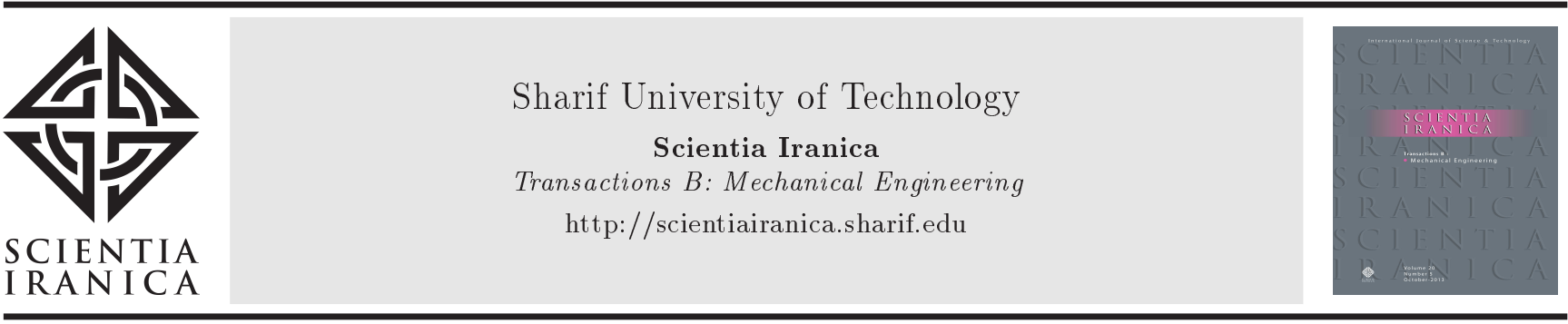

Research Note

\title{
Sensitivity analysis of the effective centrifugal pump parameters using the EFAST method
}

\author{
H. Safikhani* \\ Department of Mechanical Engineering, Faculty of Engineering, Arak University, Arak, 38156-88349, Iran.
}

Received 24 November 2016; received in revised form 30 September 2017; accepted 18 December 2017

\section{KEYWORDS}

Sensitivity analysis; EFAST method; Centrifugal pumps; Required NPSH.

\begin{abstract}
In the present study, effective parameters of centrifugal pumps are investigated using the EFAST Sensitivity Analysis (SA) method. The SA is performed using GMDHtype Artificial Neural Networks (ANN) which are based on validated numerical data of flow field in centrifugal pumps. There are four design variables: leading edge angle of blades on hub section ( $\beta_{1 \text { hub }}$ ), leading edge angle of blades on shroud section ( $\left.\beta_{1 \text { shroud }}\right)$, trailing edge angle of blades $\left(\beta_{2}\right)$, and the stagger angle of blades on mid span $\left(\gamma_{\text {mid }}\right)$. There are two objective functions: efficiency $(\eta)$ and the required NPSH of impeller. The results show that, among design variables, $\beta_{2}$ has the highest effect on variations of $\eta(46 \%)$ and NPSH (45\%). Except $\beta_{2}, \beta_{1 \text { hub }}$, and $\gamma_{\text {mid }}$ have the highest effect on NPSH (33\%) and $\eta(28 \%)$, respectively. The effects of all of the design variables on objective functions are shown in the results.
\end{abstract}

(C) 2019 Sharif University of Technology. All rights reserved.

\section{Introduction}

Centrifugal pumps are a group of turbo machines used industrially in large scales. In recent years, several researchers have investigated different aspects of such pumps. Demeulenaere et al. [1] investigated an optimization process on centrifugal pumps using fine/design 3D environment of Numeca software and genetic algorithms. They tried to increase efficiency and head and decrease the NPSHr at two different flow rates; finally, they showed that the new blade geometry should have more curvature in the camber line definition. Nariman-zadeh et al. [2] presented a multi-objective optimization process on centrifugal pumps and suggested four optimal points for a designer to select. They tried to increase the hydraulic efficiency and head and decrease the input power. They did not

* Tel.: +9886 32625726; Fax: +98 8632625001.

E-mail address: h-safikhani@araku.ac.ir use CFD in their simulation and just used the analytical equations for hydraulic efficiency, head and the input power. Safikhani et al. [3] investigated a multiobjective optimization process on centrifugal pumps. Combining CFD, GMDH-type neural networks, and NSGA II algorithm, they presented the Pareto front for centrifugal pumps.

Korakianitis et al. [4] developed specific speed versus specific diameter graphs suitable for the design and optimization of these smaller centrifugal pumps concentrating in dimensions suitable for Ventricular Assist Devices (VADs) and Mechanical Circulatory Support (MCS) devices. A combination of experimental and numerical techniques was used to measure and analyze the performances of 100 optimized pumps designed for this application. The data were presented in the traditional Cordier diagram of non-dimensional specific speed versus specific diameter. Using these data, nine efficient designs were selected to be manufactured and tested in different operating conditions of flow, pressure, and rotational speed. The non-dimensional results presented in this article enable preliminary 
design of centrifugal pumps for VADs and MCS devices. Wang et al. [5] proposed a method to optimize the design of a typical multi-stage centrifugal pump based on Energy Loss Model and Computational Fluid Dynamics (ELM/CFD). Wang et al. [6] improved the efficiency of a centrifugal pump using optimization of a vanned diffuser. The steady simulations were carried out by solving the three-dimensional Reynolds-averaged Navier-Stokes equations with a shear stress transport turbulence model. Finally, the efficiency of the optimal pump increased by $8.65 \%$, compared with the original scheme. The velocity distributions in the diffuser inlet and volute improved and became more uniform. The total pressure in the diffuser and volute of the optimal pump was higher than that of the original pump. Zhao et al. [7] described the shape optimization of a low specific speed centrifugal pump at the design point. Some other researchers have also done some studies on the optimization of different engineering elements [8-13].

In centrifugal pumps, there are many geometrical parameters; through a sensitivity analysis, the effective parameters should be defined. Sensitivity analysis refers to the study of "how uncertainty in model output (numerical and non-numerical) can be classified into different sources of uncertainty in model input factors" [14]. Saltelli et al. [15] classified the sensitivity analysis methods into two groups: local and general. The local sensitivity analysis methods analyze the response of model output(s) by changing one of the parameters and maintaining the other parameters at central values, while the general sensitivity analysis methods investigate the general response of model output(s) (averaged over the variation of all the parameters) by searching a finite (or infinite) region. Although the local sensitivity analysis method is simple to use, it just analyzes one point at a moment; thus, nowadays, the general sensitivity analysis methods are preferred to the local ones.

As was mentioned, sensitivity analysis can specify the sensitive and insensitive parameters of a model. In this regard, Korayem et al. [16] investigated the use of different contact models in the AFM-based manipulation of biological cells in bio-environments. They employed the Sobol method to analyze the sensitivity of the modeling parameters of four contact mechanic models (PT, Hertz, DMT, and JKR). Hertz model is very sensitive to the Young's modulus, and the sensitivity of the adhesion energy in this model is zero (Hertz model disregards the effect of adhesion energy). Contrary to Hertz model, the other three models are highly sensitive to the adhesion energy as well as the elasticity modulus. All the models show little sensitivity to the parameters of particle radius and Poisson's ratio.

Based on our information, no sensitivity analysis research has been carried out so far on centrifugal pumps. Therefore, sensitivity analysis is investigated in the present study using the EFAST method.

\section{Defining the design variables}

To parameterize the camber line curve, the simple Bezier method is used. Schematically, definition of a simple Bezier method is shown in Figure 1. The design variables in this method are leading edge angle of blades on hub section ( $\beta_{1 \text { hub }}$ ), leading edge angle of blades on shroud section ( $\beta_{1}$ shroud), trailing edge angle of blades $\left(\beta_{2}\right)$, and the stagger angle of blades on mid span $\left(\gamma_{\text {mid }}\right)$. In the present paper, three sections are defined in the blades: the first section on hub, the second on shroud, and the third on the middle plane of hub and shroud, as shown in Figure 2. It is supposed that $\beta_{2}$ is the same in the three defined sections of blade. This problem is mathematically given by:

$$
\beta_{2 \text { hub }}=\beta_{2 \text { shroud }}=\beta_{2 \text { midspan }}=\text { Design Variable. }
$$

Moreover, $\beta_{1}$ at mid span is equal to the average of $\beta_{1}$ at hub and shroud sections:

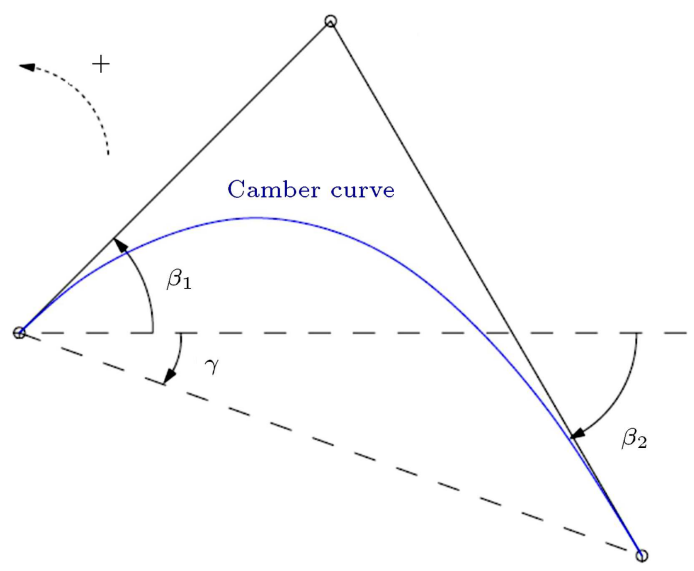

Figure 1. Blade camber line parameterization using simple Bezier method.

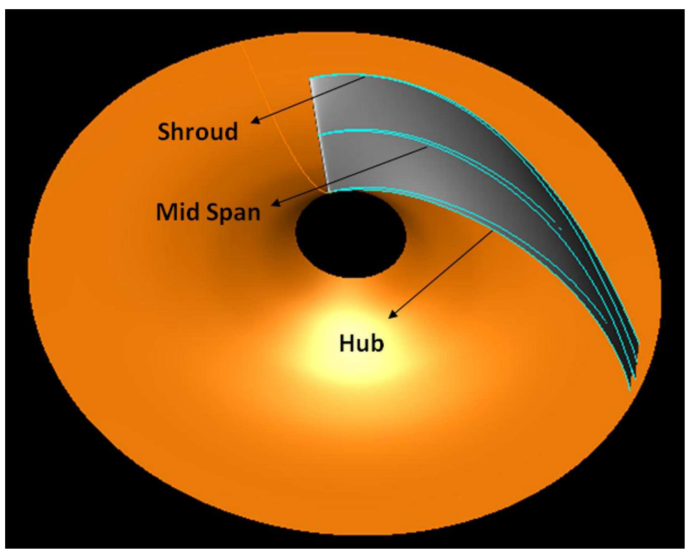

Figure 2. Defining three sections on centrifugal pumps blade. 
Table 1. Design variables and their range of variations.

\begin{tabular}{ccc}
\hline Design variable & From $(\mathbf{d e g})$ & To $(\mathbf{d e g})$ \\
\hline$\beta_{1 \text { hub }}$ & 0 & 30 \\
$\beta_{1 \text { shroud }}$ & 60 & 89 \\
$\beta_{2}$ & 40 & 60 \\
$\gamma_{\text {midspan }}$ & 30 & 70 \\
\hline
\end{tabular}

Table 2. The operating conditions in the simulations.

\begin{tabular}{lc}
\hline \multicolumn{1}{c}{ Parameter } & Value \\
\hline Number of blades & 7 \\
Rotational velocity $(\mathrm{rpm})$ & 2900 \\
Mass flow $(\mathrm{kg} / \mathrm{s})$ & $24.7(\mathrm{BEP})$ \\
Outlet static pressure (atm) & 3.2 \\
\hline
\end{tabular}

$$
\beta_{1 \text { midspan }}=\frac{\beta_{1 \text { hub }}+\beta_{1 \text { shroud }}}{2}
$$

So, there are four independent design variables: $\beta_{1 \text { hub, }}$

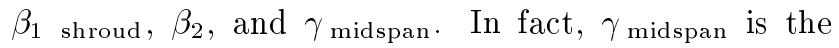
average $\gamma$ of three sections. Design variables and their range of variations are shown in Table 1 . The sensitivity analysis in the present paper is performed using the GMDH-type Artificial Neural Network (ANN) models and CFD data, presented in [3].

\section{CFD and GMDH-type ANN models}

The Sensitivity Analysis (SA) presented in this paper is performed using GMDH-type Artificial Neural Networks (ANN), which are based on validated numerical data of flow field in centrifugal pumps. The details of numerical modeling and GMDH polynomials are presented in [3]. Some operating conditions are shown in Table 2; moreover, a sample of grid generation and pressure contour in numerical simulations are shown in Figures 3 and 4, respectively.

\section{Sensitivity analysis methods}

An area of general sensitivity analysis methods that has attracted more attention is the variance-based methods. In these methods, the sensitivity index is computed as the share of each parameter in the overall output variance of the model. The general sensitivity analysis methods are implemented in four steps: (1) defining the inputs and the type of distribution of each input, (2) generating the samples for the input values, (3) computing the model's output for each set of input samples, and (4) determining the effect of each input factor on the output [17]. In this section, the variance-based sensitivity analysis methods are reviewed. The variance-based general sensitivity analysis approaches can be used to obtain the first-

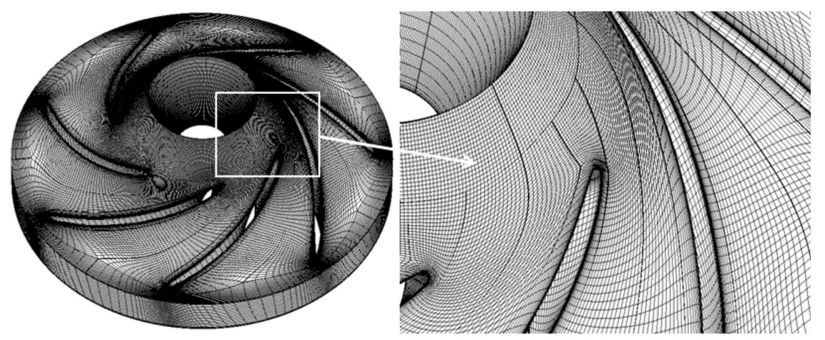

Figure 3. A sample of CFD structured grid generation for centrifugal pumps.

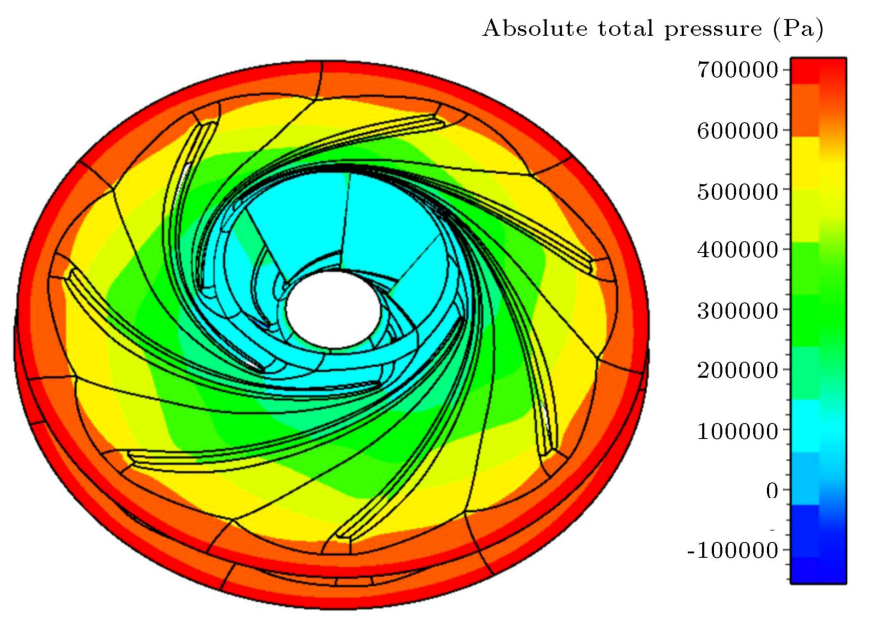

Figure 4. A sample of pressure contour in CFD simulations of centrifugal pumps.

order and the second-order effects (which include the interaction between other parameters) [18].

The Sobol method [19] is a model-independent general sensitivity analysis method which is based on variance analysis. This method can be used for nonlinear and non-uniform functions and models. For the model defined by function $Y=f(X)$, where $Y$ is the model output and $X\left(x_{1}, x_{2}, \ldots, x_{n}\right)$ is the vector of input parameters, Sobol suggested to decompose function $f$ into summands of increasing dimensionality, where the integral of each term over its own input variables is zero. Sobol showed that, when all the inputs are perpendicular to one another, this resolution is unique, and the output variance of the model $(V)$ is the set of variances of each resolved term [19]:

$$
V(Y)=\sum_{i=1}^{n} V_{i}+\sum_{i \leq j \leq n}^{n} V_{i j}+\ldots+V_{1 \ldots n} .
$$

In Eq. (3), $V_{i}$ denotes the first-order effect for each input factor $x_{i}\left(V_{i}=V\left[E\left(Y \mid x_{i}\right)\right]\right)$, and $V_{i j}\left(V_{i j}=\right.$ $\left.V\left[E\left(Y \mid x_{i}, x_{j}\right)\right]-V_{i}-V_{j}\right)$ to $V_{1, \ldots, n}$ indicate the interactions between $n$ factors. Therefore, the shares allocated to parameters and interactions of parameters can be determined from the total output variance. The sensitivity index is obtained as the ratio of each order's 
variance to the total variance $\left(S_{i}=V_{i} / V\right.$ denotes the first-order sensitivity index, $S_{i j}=V_{i j} / V$ represents the second-order sensitivity index, and so on). The total sensitivity index (i.e., the overall effect of each parameter) is obtained as the summand of all the orders of sensitivity index for that parameter [19]:

$$
S_{T i}=S_{i}+\sum_{i \neq j} S_{i j}+\cdots
$$

The EFAST method was presented by Cukier et al. [20] and was later improved by Saltelli et al. [21]. Like the Sobol method, this approach is also based on variance and is independent of any assumption of linearity and uniformity between inputs and output(s). Contrary to the Sobol method, which uses multi-dimensional integrals to obtain the total variance and the partial variances, this method converts the multi-dimensional integrals to one-dimensional ones by defining a transfer function and simplifies the procedure for the calculation of sensitivity indexes.

The EFAST method searches the $n$-dimensional space of the input factors (unit hypercube $K^{n}$ ) using a search curve defined by a set of parametric equations [21]:

$$
x_{i}=\frac{1}{2}+\frac{1}{\pi} \arcsin \left(\sin \left(\omega_{i} s+\varphi_{i}\right)\right)
$$

where $\omega_{i}(i=1,2, \ldots, n)$ is the frequency related to factor $x_{i}, s$ is a variable that changes from $-\pi$ to $+\pi$, and $\varphi_{i}$ specifies the starting point of the curve. The output variance of the model is approximated by means of Fourier analysis:

$$
\begin{aligned}
V(Y) & =\frac{1}{2 \pi} \int_{-\pi}^{\pi} f^{2}(s) d s-\left[\frac{1}{2 \pi} \int_{-\pi}^{\pi} f(s) d s\right]^{2} \\
& \approx \sum_{j=-\infty}^{\infty}\left(A_{j}^{2}+B_{j}^{2}\right)-\left(A_{0}^{2}+B_{0}^{2}\right) \\
& \approx 2 \sum_{j=1}^{N}\left(A_{j}^{2}+B_{j}^{2}\right) .
\end{aligned}
$$

In the above relation, the following:

$$
f(s)=f\left(G_{1}\left(\sin \left(\omega_{1} s\right)\right), G_{2}\left(\sin \left(\omega_{2} s\right)\right), \ldots, G_{n}\left(\sin \left(\omega_{n} s\right)\right)\right),
$$

and $G(s)$ represent the transfer functions, and $A_{j}$ and $B_{j}$ are the Fourier coefficients, i.e.:

$$
\begin{aligned}
& A_{j}=\frac{1}{2 \pi} \int_{-\pi}^{\pi} f(s) \cos (j s) d s, \\
& B_{j}=\frac{1}{2 \pi} \int_{-\pi}^{\pi} f(s) \sin (j s) d s .
\end{aligned}
$$

By calculating the Fourier coefficients for the basic frequency $\left(\omega_{i}\right)$ and their higher harmonics $\left(p \omega_{i}\right)$, the partial first-order input variance $\left(x_{i}\right)$ can be obtained.

$$
V_{i}=\sum_{p \in Z^{0}}\left(A_{p \omega_{i}}^{2}+B_{p \omega_{i}}^{2}\right)=2 \sum_{p=1}^{\infty}\left(A_{p \omega_{i}}^{2}+B_{p \omega_{i}}^{2}\right) .
$$

In addition, similar to the Sobol method, the ratio of the first-order partial variance to total variance is used to compute the main sensitivity index. The total sensitivity index is obtained from Eq. (8) [22]:

$$
S T_{i}=1-\frac{V_{-i}}{V}
$$

Variance $V_{-i}$ is obtained by changing all the parameters except parameter $x_{i}$.

The Sobol method employs the Monte Carlo integral to obtain each partial variance; in comparison with the EFAST method, it does not use a transfer function, which is why it has low computational efficiency. Algorithm of sensitivity analysis is shown in Figure 5.

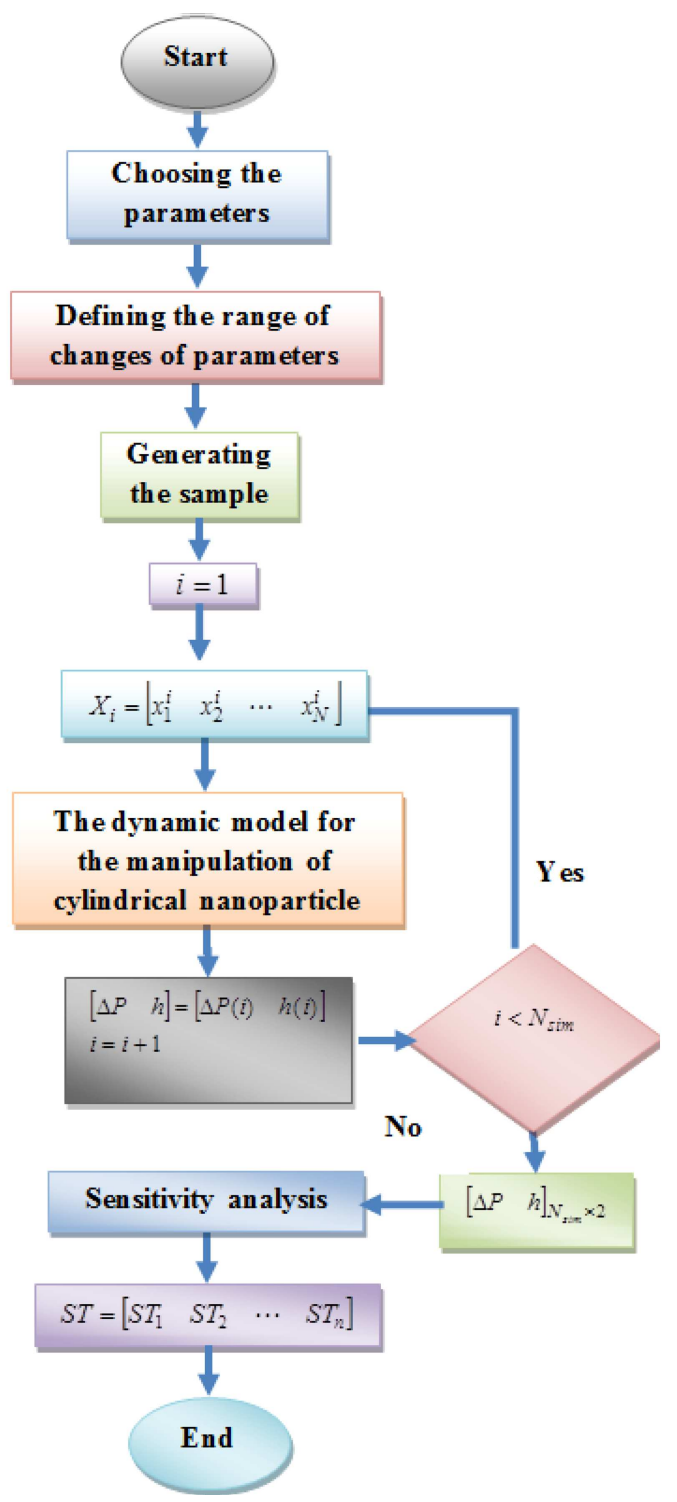

Figure 5. Algorithm of sensitivity analysis. 


\section{Results of sensitivity analysis}

The results of sensitivity analysis for efficiency $(\eta)$ and the Net Positive Suction Head (NPSH) in centrifugal pumps are presented in this section. Employing the EFAST method, the sensitivity of four parameters, i.e., leading edge angle of blades on hub section $\left(\beta_{1}\right.$ hub $)$, leading edge angle of blades on shroud section ( $\left.\beta_{1 \text { shroud }}\right)$, trailing edge angle of blades $\left(\beta_{2}\right)$, and the stagger angle of blades on mid span $\left(\gamma_{\text {mid }}\right)$, have been explored for $\eta$ and NPSH. Table 1 shows the intervals of changes of the investigated parameters.

Figure 6(a) shows the changes of $\eta$ with $\beta_{1}$ hub and indicates that with the increase of this parameter, the $\eta$ diminishes with a sharp slope. As observed in this figure, at low values of $\beta_{1}$ hub, sensitivity is smaller, and with the increase of $\beta_{1}$ hub, the slope of the diagram becomes greater. So, by considering the results that indicate the effect of this parameter on $\eta$, the proper values for this parameter can be selected. As shown in Figure 6(b), with the increase in $\beta_{2}, \eta$ also diminishes with a very sharp slope. Therefore, the first most sensitive parameter is $\beta_{2}$.

The other investigated parameter is $\beta_{1 \text { shroud; }}$ considering a near-zero slope for the diagram showing the changes of $\eta$ versus $\beta_{1}$ shroud (Figure 6(c)), this parameter is not considered to be a sensitive parameter for $\eta$, and choosing different values for this parameter from its range of changes does not lead to a tangible change in $\eta$ values. As Figure 6(d) demonstrates, the diagram which shows the changes of $\eta$ versus $\gamma_{\text {mid }}$ is selected, indicating that with the increase of this parameter, first, $\eta$ decreases and, then, increases.

The changes of the NPSH with $\beta_{1}$ hub are shown in Figure $7(\mathrm{a})$. With the increase of $\beta_{1}$ hub, NPSH diminishes with a very sharp slope. As is observed in this figure, at low values of $\beta_{1 \text { hub }}$, sensitivity is smaller, and with the increase of $\beta_{1}$ hub, the slope of the diagram becomes greater. Thus, by considering the results that indicate the effect of this parameter on the NPSH, the proper values for this parameter can be selected. Another sensitive parameter among the parameters is $\beta_{2}$. According to Figure $7(\mathrm{~b})$, with the increase of this parameter, NPSH also increases with a sharp slope.

The other investigated parameter is $\beta_{1 \text { shroud; }}$ considering a near-zero slope for the diagram showing

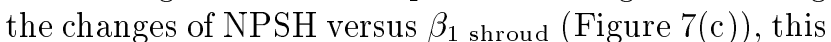
parameter is not considered to be a sensitive parameter for the NPSH, and choosing different values for this parameter from its range of changes does not lead to a tangible change in the NPSH values. Another sensitive parameter among the input parameters is $\gamma_{\text {mid }}$. According to Figure $7(\mathrm{~d})$, with the increase of this parameter, first, the NPSH also increases and, then, decreases.

Figure 8 indicates more accurate analysis of the results obtained by the EFAST sensitivity analysis method. According to Figure 8, among the aforementioned four parameters, as expected, $\beta_{2}$ (with a sensitivity index of $46 \%$ ), $\gamma_{\text {mid }}$ (with a sensitivity

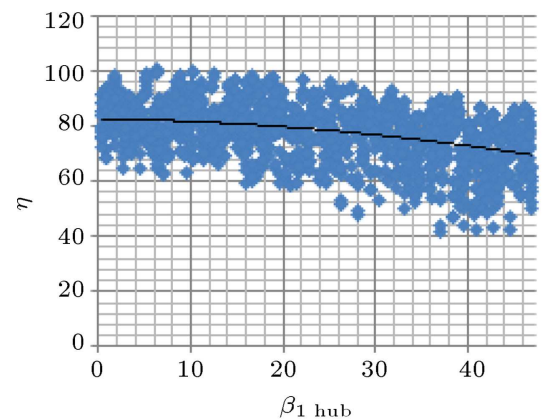

(a)

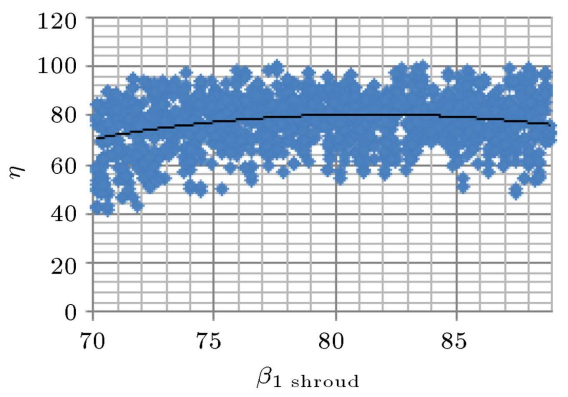

(c)

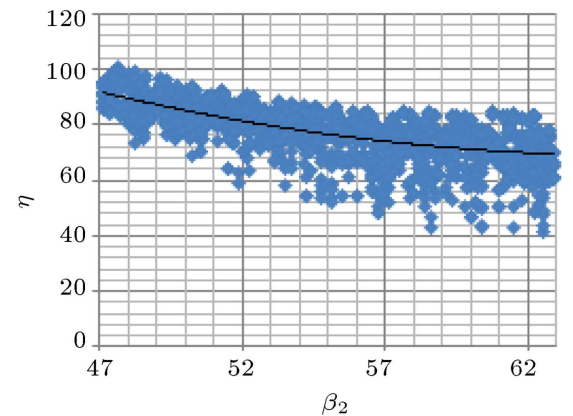

(b)

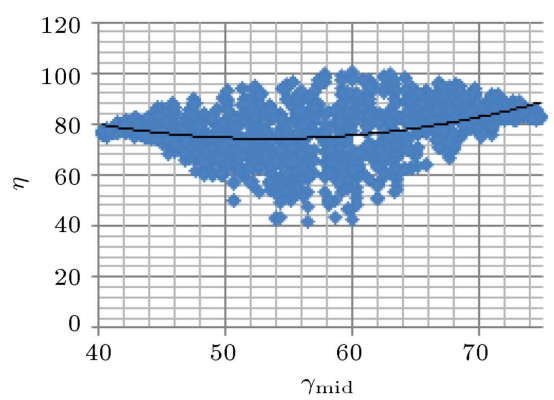

(d)

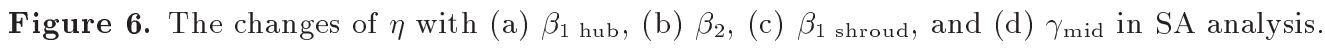




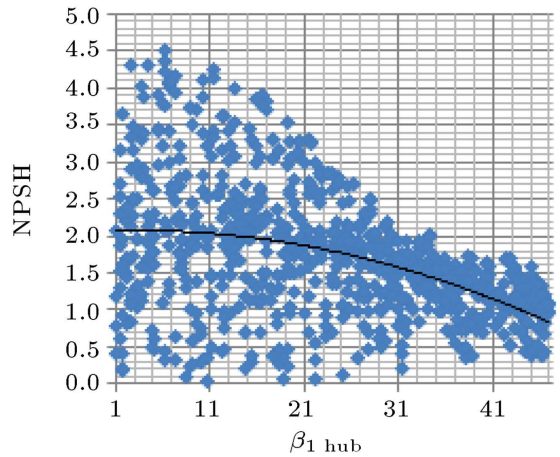

(a)

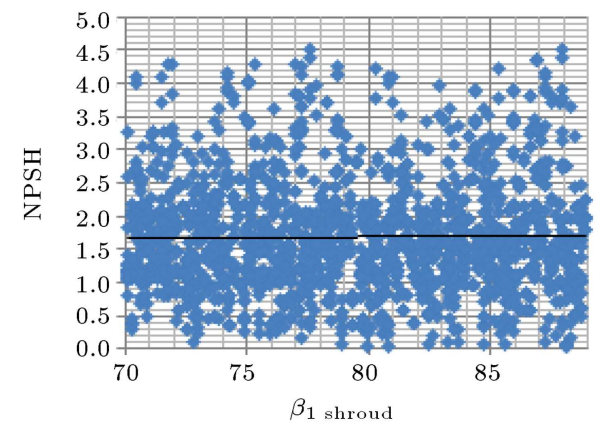

(c)

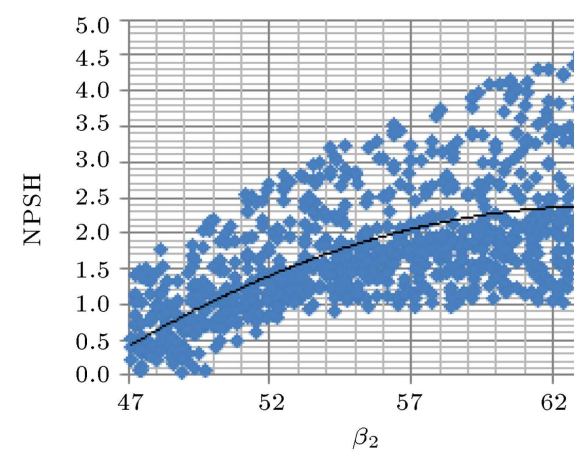

(b)

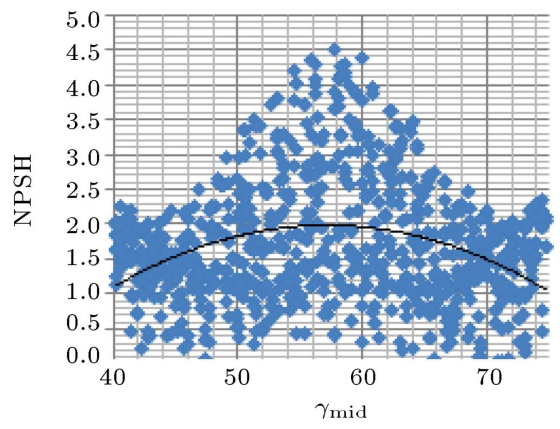

(d)

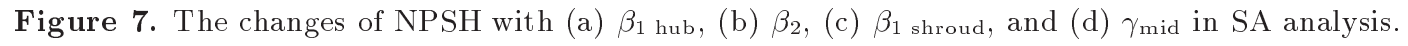
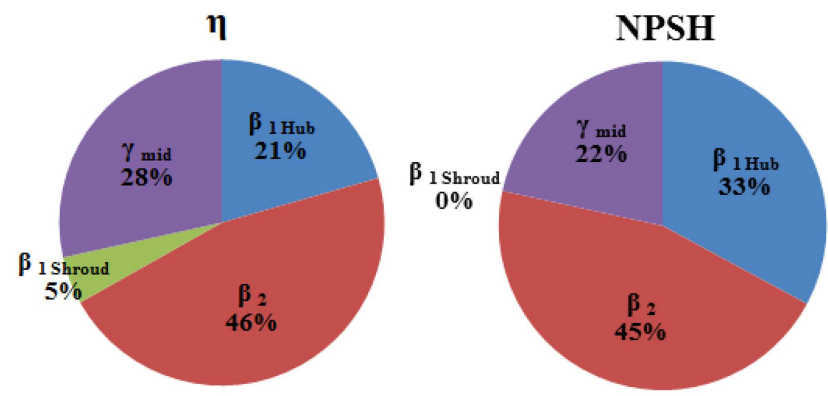

Figure 8. Percent sensitivity of input parameter changes in the $\eta$ and NPSH.

index of $28 \%$ ), and $\beta_{1}$ hub (with a sensitivity index of $21 \%$ ) are the most significant sensitivity parameters in $\eta$. Further, according to Figure $8, \beta_{2}$ (with $45 \%$ sensitivity) is the most important parameter; the parameters of $\beta_{1}$ hub (with $33 \%$ sensitivity) and $\gamma_{\text {mid }}$ (with $22 \%$ sensitivity) are, respectively, the other effective parameters in NPSH.

\section{Conclusion}

The effective parameters of centrifugal pumps were investigated using the EFAST sensitivity analysis method. The SA was performed using GMDH-type ANN based on validated numerical data of flow field in centrifugal pumps. There were four design variables: $\beta_{1 \text { hub }}, \beta_{1 \text { shroud }}, \beta_{2}$, and the stagger angle of blades on mid span $\gamma_{\text {mid }}$. There were two objective functions: $\eta$ and the required NPSH of impeller. The results show that, among design variables, $\beta_{2}$ has the highest effect on variations of $\eta(46 \%)$ and NPSH (45\%). Except $\beta_{2}, \beta_{1}$ hub and $\gamma_{\text {mid }}$ have the highest effect on NPSH $(33 \%)$ and $\eta(28 \%)$, respectively. The effects of all of the design variables on objective functions were shown in the results (Figure 8).

\section{References}

1. Demeulenaere, A., Purwanto, A., Ligout, A., Hirsch, C., Dijkers, R., and Visser, F. "Design and optimization of an industrial pump: Application of genetic algorithm and neural network", Proceedings of Insert Conference Abbreviation, ASME Fluid Engineering Summer Conference, Houston, Texas (2005).

2. Nariman-zadeh, N., Amanifard, N., Hajiloo, A., Ghalandari, P., and Hoseinpoor, B. "Multi-objective pareto optimization of centrifugal pumps using genetic algorithms", Proceedings of 11th WSEAS International Conference on Computers, Crete Island, Greece (2007).

3. Safikhani, H., Khalkhali, A., and Farajpoor, M. "Pareto based multi-objective optimization of centrifugal pumps using CFD, neural networks and genetic algorithms", Engineering Applications of Computational Fluid Mechanics, 5, pp. 37-48 (2011).

4. Korakianitis, T., Rezaienia, M., Gordon, P., Rahideh, A., Rothman, T., and Mozafari, S. "Optimization of centrifugal pump characteristic dimensions for me- 
chanical circulatory support devices", ASAIO Journal, 62(5), pp. 545-551 (2016).

5. Wang, C., Shi, W., Wang, X., Jiang, X., Yang, Y., Li, W., and Zhou, L. "Optimal design of multistage centrifugal pump based on the combined energy loss model and computational fluid dynamics", Applied Energy, 187, pp. 10-26 (2017).

6. Wang, W., Shouqi, Y., and Ji, P. "Optimization of the diffuser in a centrifugal pump by combining response surface method with multi-island genetic algorithm", Proceedings of the Institution of Mechanical Engineers, Part E: Journal of Process Mechanical Engineering, 231(2), pp. 13-24 (2017).

7. Zhao, A., Lai, Z., Wu, P., Cao, L., and Wu, D. "Multiobjective optimization of a low specific speed centrifugal pump using an evolutionary algorithm", Engineering Optimization, 48(7), pp. 1251-1274 (2016).

8. Yun, X., Lei, T., and Shuliang, C. "Multiparameter and multiobjective optimization design of centrifugal pump based on orthogonal method", Proceedings of the Institution of Mechanical Engineers, Part C: Journal of Mechanical Engineering Science, 231(14), pp. 19-28 (2017).

9. Safikhani, H. "Modeling and multi-objective Pareto optimization of new cyclone separators using CFD, ANNs and NSGA II algorithm", Advanced Powder Technology, 27(5), pp. 2277-2284 (2016).

10. Safikhani, H. and Dolatabadi, H. "Multi-objective optimization of cooling of a stack of vertical minichannels and conventional channels subjected to natural convection", Applied Thermal Engineering, 96, pp. 144-150 (2016).

11. Damavandi, M.D., Forouzanmehr, M., and Safikhani, H. "Modeling and pareto based multi-objective optimization of wavy fin-and-elliptical tube heat exchangers using CFD and NSGA-II algorithm", Applied Thermal Engineering, 111, pp. 325-339 (2017).

12. Sadafi, M.H., Hosseini, R., Safikhani, H., Bagheri, A., and Mahmoodabadi, M.J. "Multi-objective optimization of solar thermal energy storage using hybrid of particle swarm optimization, multiple crossover and mutation operator", International Journal of Engineering, 24(3), pp. 366-376 (2011).

13. Safikhani, H. and Eiamsa-ard, S. "Pareto based multiobjective optimization of turbulent heat transfer flow in helically corrugated tubes", Applied Thermal Engineering, 95, pp. 275-280 (2016).
14. Saltelli, A. and Sobol, M. "About the use of rank transformation in sensitivity analysis of model output", Reliability Engineering \& System Safety, 50, pp. 225-239 (1995).

15. Saltelli, A., Chan, K., and Scott, E., Sensitivity Analysis Wiley Series in Probability and Statistics, Willey, New York (2000).

16. Korayem, M., Rastegar, Z., and Taheri, M. "Sensitivity analysis of nano-contact mechanics models in manipulation of biological cell", Nanoscience and Nanotechnology, 2, pp. 49-56 (2012).

17. Tong, C. "Self-validated variance-based methods for sensitivity analysis of model outputs", Reliability Engineering \& System Safety, 95, pp. 301-309 (2010).

18. Nossent, J., Elsen, P., and Bauwens, W. "Sobol' sensitivity analysis of a complex environmental model", Environmental Modelling \& Software, 26, pp. 15151525 (2011).

19. Sobol, I.M. "Sensitivity estimates for nonlinear mathematical models", Mathematical Modeling and Computational Experiments, 14, pp. 407-414 (1993).

20. Cukier, R., Levine, H., and Shuler, K. "Nonlinear sensitivity analysis of multiparameter model systems", Journal of Computational Physics, 26, pp. 1-42 (1978).

21. Saltelli, A., Tarantola, S., and Chan, K.-S. "A quantitative model-independent method for global sensitivity analysis of model output", Technometrics, 41, pp. 3956 (1999).

22. Homma, T. and Saltelli, A. "Importance measures in global sensitivity analysis of nonlinear models", Reliability Engineering \& System Safety, 52, pp. 1-17 (1996).

\section{Biography}

Hamed Safikhani is an Assistant Professor of Mechanical Engineering at the Arak University, I.R. Iran. He received his $\mathrm{PhD}$ from the Amirkabir University of Technology in 2014. He is one of the members of "The Promised SORAYYA Technologist" sciencebased industries in I.R. Iran. He has co-authored more than 40 publications in high-quality journals and conferences. His research interests include air pollution numerical modelling, HVAC systems, energy, two-phase and single-phase convective heat transfers in macro-micro and nanoscales. 